DOI: https://doi.org/10.11144/Javeriana.umed60-4.malt

\title{
Maltrato a las personas mayores: una revisión narrativa
}

\author{
Elder Abuse: A Narrative Review
}

Recepción: 11/03/2019 | Aceptación: 13/05/2019

\author{
Laura Margarita Forero Borda ${ }^{\mathrm{a}}$ \\ Médica residente de Geriatría, Facultad de Medicina, \\ Pontificia Universidad Javeriana, Bogotá, Colombia \\ Saith Hoyos Porto \\ Médico geriatra, Hospital Universitario San Ignacio, \\ Bogotá, Colombia \\ Viviana Buitrago Martínez \\ Trabajadora social del Hospital Universitario San \\ Ignacio, Bogotá, Colombia \\ Rodrigo Alberto Heredia Ramírez \\ Médico geriatra, Hospital Universitario San \\ Ignacio. Profesor, Facultad de Medicina, Pontificia \\ Universidad Javeriana, Bogotá, Colombia
}

a Correspondencia: la-forero@javeriana.edu.co

\section{RESUMEN}

El maltrato a las personas mayores es un tema de interés creciente, tanto así que se considera un problema de salud pública. Hay particularidades que hacen del entorno colombiano un ambiente propicio para generar situaciones de maltrato: la exposición a la "violencia a gran escala", la diferencia de género y el cambio de la pirámide poblacional. Según el estudio SABE Colombia, el 12,9\% de las personas mayores informó haber sufrido maltrato, del cual el más común es el psicológico, seguido por el físico, el financiero y el sexual. A pesar de que el Estado reconoce al adulto mayor como "sujeto de especial protección", aún hay desconocimiento por parte de los profesionales de la salud a la hora de enfrentar estos casos. Este debería ser un tema de obligatoriedad moral, por lo que se debe abordar a profundidad para así identificarlo oportunamente y proponer estrategias de prevención de una manera contextualizada. Esta revisión de la literatura tiene por objetivos sintetizar la información epidemiológica disponible, los factores de riesgo y las estrategias de evaluación y plantear las rutas de atención idóneas asentadas en los aspectos legales alrededor del maltrato hacia los ancianos en Colombia.

\section{Palabras clave}

maltrato al anciano; negligencia; cuidador.

\section{ABSTRACT}

Elder abuse is a topic of growing interest and it is considered as a public health problem. The Colombian context has built an ideal climate generating situations of abuse: the exposure to "violence on a large scale", the gender gap and the change of the population pyramid. According to the SABE Colombia, $12.9 \%$ of the elderly reported having suffered abuse, the most common is psychological abuse, followed by the physical, financial and sexual. Although the Colombian State recognizes the elderly
Cómo citar: Forero Borda LM, Hoyos Porto S, Buitrago Martínez V, Heredia Ramírez RA. Maltrato a las personas mayores: una revisión narrativa. Univ. Med. 2019;60(4). https:// doi.org/10.11144/Javeriana.umed60-4.malt 
as a special subject of protection, perhaps there is still ignorance of the health professionals. This should be a moral obligation for the medical community, so it must be addressed in depth in order to identify cases in time and propose prevention. This review of the literature aims to synthesize the available epidemiological information, clinical evidence and legal aspects of elder abuse in the Colombian context and finally propose routes of attention with the legal aspects surrounding the abuse.

Keywords

elder abuse; negligence; caregiver.

\section{Introducción}

La trasformación de la pirámide poblacional ha develado un problema que, con seguridad, existía $(1,2,3)$, pero que se ha agravado no solo por la mayor proporción de ancianos en todo el mundo, sino por la inversión de sus roles. Se cree que los ancianos han pasado de una posición de respeto en la sociedad humana, desde la cual concentraban la sabiduría y el poder económico y político, hacia otra versión menos favorable en la sociedad industrializada. Lamentablemente, existe una representación negativa de "carga" de la persona mayor, tanto para los sistemas de seguridad social como para quienes asumen el rol de cuidadores $(4,5)$. Entre las posibles causas de construcción de este imaginario están: la ruptura de la familia como unidad básica de la sociedad, las nuevas formas de organización y vinculación con tendencia hacia un concepto más egocéntrico y hedonista de la vida (6), lo cual está sumado a los avances en medicina que pueden prolongar la vida hasta altos niveles de dependencia. Estas circunstancias han creado un ambiente propicio para que en el mundo el maltrato al anciano se haya convertido en un problema de salud pública que se debe investigar y comprender en el ámbito de cada país, cada cultura y cada clase social.

La primeras publicaciones alrededor del maltrato hacia la persona mayor datan de los años ochenta en Gran Bretaña, Estados Unidos y Canadá $(7,8)$. A medida que se consolidaron las instituciones científicas de geriatría y gerontología, el interés por su estudio fue cobrando más fuerza. Así, en España, en la Primera Conferencia de Consenso sobre el Anciano Maltratado, en 1996, se definió como:

[...] todo acto u omisión sufrido por personas de 65 años o más, que vulnera la integridad física, psíquica, sexual, y económica, el principio de autonomía, o un derecho fundamental del individuo; que es percibido por este o constatado objetivamente, con independencia de la intencionalidad y del medio donde ocurra (familiar, comunidad e instituciones). (9)

Posteriormente, la Declaración de Toronto, a través de Red Internacional de Prevención del Abuso y Maltrato en la Vejez (INPEA) y la Organización Mundial de la Salud (OMS), lo definió como "[...] la acción única o repetida, o la falta de la respuesta apropiada, que ocurre dentro de cualquier relación donde exista una expectativa de confianza y la cual produzca daño o angustia a una persona adulta..." $(10,11)$.

En Colombia, la definición aceptada por el Instituto de Medicina Legal acoge dentro del concepto de violencia familiar "cualquier forma de maltrato, ya sea físico, psicológico o sexual, que tiene lugar entre los miembros de una familia" (12). Esta se cita dentro del capítulo de "Comportamiento de las lesiones por violencia intrafamiliar"; sin embargo, en esta revisión se retoma el término maltrato, ya que posiciona al anciano no solamente dentro de la dinámica familiar, sino también como parte de la comunidad. Una consideración relevante en cuanto la historia de vida del colombiano es la influencia de la "violencia a gran escala" como un cotidiano que ocupa los titulares de los medios de comunicación lo que al parecer ha aumentado el umbral de respuesta a las manifestaciones de "violencia menor", como la violencia intrafamiliar y el maltrato hacia la persona mayor (1).

Esta revisión de la literatura tiene como objetivo sintetizar la información epidemiológica disponible, la evidencia clínica y los aspectos legales del maltrato a los ancianos en el contexto colombiano, a fin de visibilizar esta problemática, identificar casos oportunamente y fortalecer las rutas de atención. 


\section{Epidemiología}

Se estima que solo un $20 \%$ de los casos es notificado, ya que pueden existir dificultades al denunciar por parte de la víctima, como miedo a represalias, ruptura de la relación con los hijos, temor al abandono, falta de conciencia sobre la gravedad de la situación, incapacidad por una demencia, no detección por profesionales debido a falta de información y dudas en la actuación $(13,14)$.

Según una revisión sistemática reciente, la prevalencia mundial del maltrato a personas mayores es del $15,7 \%$ y uno de cada seis experimentó alguna forma de maltrato en el último año. En la misma revisión se citan cifras de prevalencia en países como Estados Unidos, donde llega a ser hasta del 10\%; Reino Unido, que oscila entre el $2 \%$ y el $6 \%$; Canadá, con el 6\%, y España, con el 3\% (15). En el caso de América Latina, por año se han registrado 38.000 casos de violencia contra los ancianos, en los que las tasas varían desde el $10 \%$ al $12 \%$.

En 2017, en Colombia, el Centro de Referencia Nacional sobre Violencia, del Instituto Nacional de Medicina Legal y Ciencias Forenses, destacó un ascenso de las lesiones no fatales contra el adulto mayor, en un $17,6 \%$. En el mismo año se realizaron 27.538 exámenes médico-legales por violencia intrafamiliar, y de estos el 7,06\% correspondía a violencia contra el adulto mayor (12).

Según la Encuesta Nacional de Salud Bienestar y Envejecimiento (SABE Colombia), el 12,9\% de las personas mayores informó haber sufrido maltrato. Se encontró mayor proporción de maltrato psicológico $(12,3 \%)$, seguido de físico $(3,4 \%)$, financiero $(1,5 \%)$ y sexual $(0,3 \%)$ (figura 1). Se registró una mayor proporción de todos los tipos de maltrato en la zona rural, a excepción del maltrato psicológico, que fue mayor en la zona urbana (figura 2). La mayor proporción de maltrato psicológico se ubicó en los estratos socioeconómicos más bajos, lo que pone en evidencia situaciones de pobreza como factor de riesgo de la población colombiana. El estrato 1 tuvo mayor proporción de maltrato físico y sexual; mientras que los estratos 5 y 6 tuvieron mayores frecuencias de maltrato financiero (figura 3). Esto también varía según el grupo de edad (figura 4).

\section{Figura 1}

Encuesta SABE: tipos de maltrato contra el anciano en Colombia

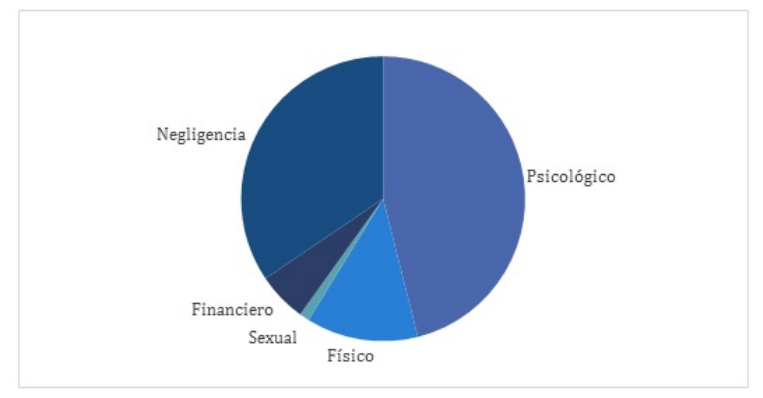

\section{Figura 2}

Encuesta SABE: distribución del tipo de maltrato según zona

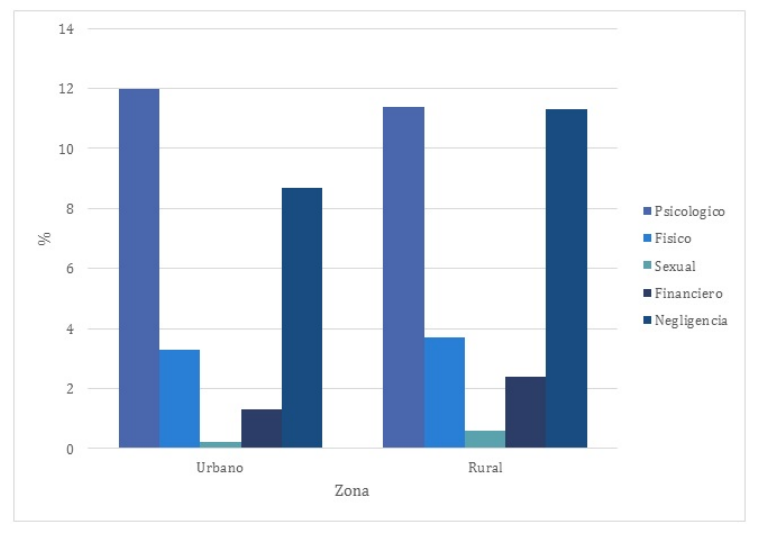

\section{Figura 3}

Encuesta SABE: distribución del tipo de maltrato según el estrato

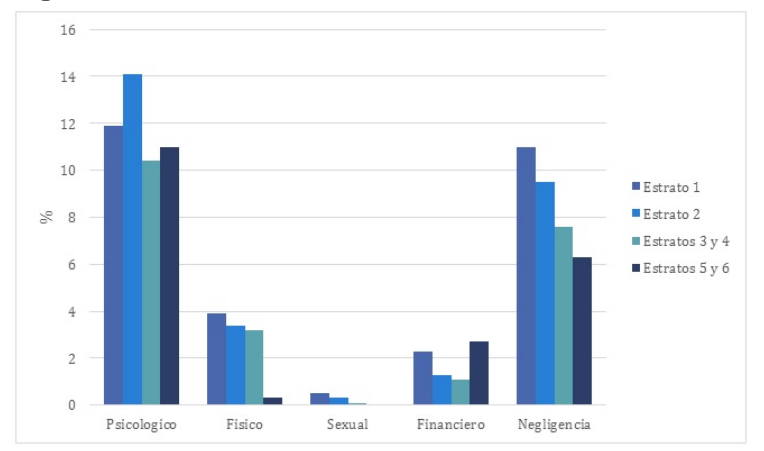




\section{Figura 4}

Encuesta SABE: distribución del tipo de maltrato según el grupo de edad

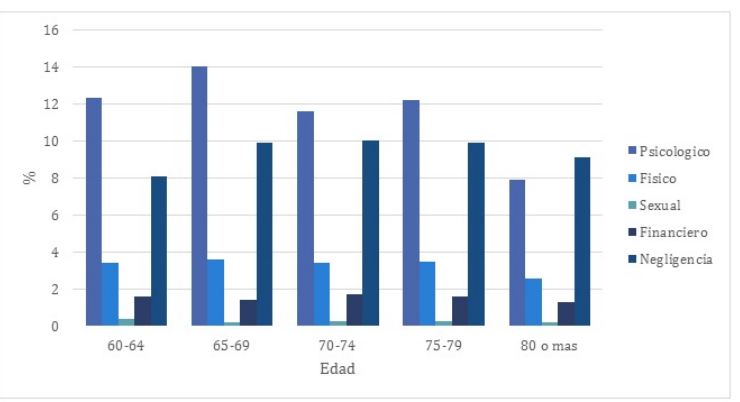

Datos más recientes del Instituto de Medicina Legal muestran que entre enero y mayo de 2018 se registraron 893 denuncias por violencia contra el adulto mayor. De estos, 485 fueron mujeres mayores de 60 años (figuras 5 y 6).

\section{Figura 5}

Denuncias por violencia contra mayores de 60 años según datos del Instituto Nacional de Medicina Legal y Ciencias Forenses

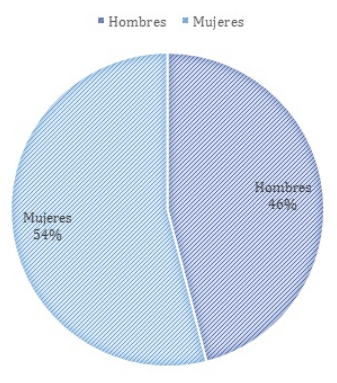

\section{Figura 6}

Encuesta SABE: distribución del tipo de maltrato según sexo

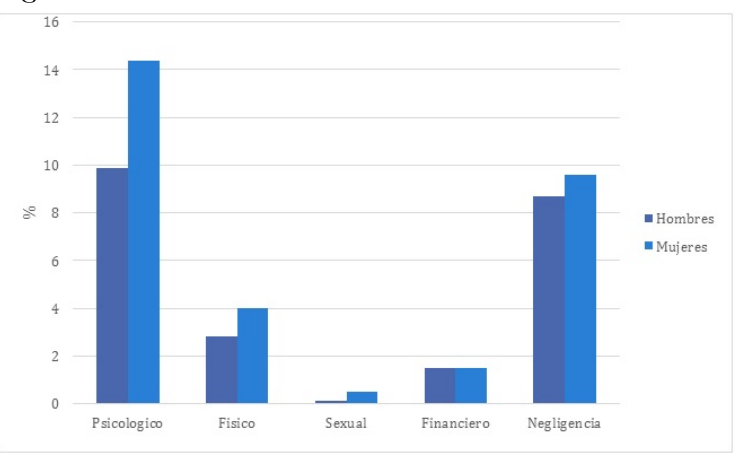

\section{Tipos de maltrato}

En la población mayor existen tipos reconocidos de maltrato: el maltrato físico, el psicológico, el económico (también llamado patrimonial o financiero), el sexual y la negligencia (11).

El maltrato psicológico es el más frecuente; tiene su origen en una relación potencialmente dañina entre el anciano y el cuidador, pues le genera al primero sentimientos de inseguridad, angustia y baja autoestima. Además, transgrede la dignidad y el respeto a la autonomía (16). En un estudio realizado en Antioquia (Colombia), el tipo de maltrato más frecuente fue el psicológico (5\%), y en otro estudio en Pasto $(5,4 \%)$, este también fue el más encontrado. Se reconoce su existencia en todos los escenarios. Un ejemplo de ello son los resultados de un estudio en el que el $81 \%$ de enfermeras domiciliarias había sido testigo de este tipo de maltrato (17). Algunas manifestaciones son el aislamiento social, la perturbación de la libertad, las amenazas de institucionalización y de abandono, las burlas, las agresiones verbales, la ridiculización, las intimidaciones, la infantilización y las humillaciones $(13,18)$.

El maltrato físico se caracteriza por el uso de la fuerza física, que deteriora la integridad corporal por medio de golpes, quemaduras, empujones, inmovilización, sujeción mecánica o administración forzada de medicación inapropiada y se pone en evidencia por la presencia de abrasiones, laceraciones, hematomas, fracturas y quemaduras. Estas manifestaciones pueden llevar a lesiones o discapacidad temporal o permanente $y$, en casos extremos, a la muerte (19). Medicina Legal, en Colombia, informa que los más perjudicados son personas de entre 60 y 64 años, siendo los hijos los principales victimarios y el factor desencadenante es la intolerancia. El mecanismo causal de la lesión es contundente y el politraumatismo es el diagnostico topográfico con más casos (12).

En cuanto a la violencia sexual, se define de igual manera para cualquier grupo de edad. La OMS la determina como: 
[...] todo acto sexual, la tentativa de consumar un acto sexual, los comentarios e insinuaciones sexuales no deseados, o las acciones para comercializar o utilizar de cualquier otro modo la sexualidad de una persona mediante coacción por otra persona, independientemente de la relación de esta con la víctima, en cualquier ámbito, incluidos el hogar, y el lugar de trabajo. (20)

La legislación colombiana la determina con similares palabras y se encuentra regulado en la Resolución 459 de 2012, en la cual se adopta el "protocolo y modelo de atención integral en salud para víctimas de violencia sexual", que incluye a poblaciones vulnerables como las personas mayores. Es importante destacar que este grupo poblacional no está preparado para los riesgos sexuales que existen, ya que, ante el estigma social, la vergüenza interiorizada y la autoculpa, minimizan los actos de violencia sexual (como las caricias, la exhibición de genitales, la masturbación, así como el sexo oral, anal o vaginal sin su consentimiento, empleando la fuerza, las amenazas y el engaño) (21). Estos actos se manifiestan expresamente o encubierto mediante gestos, palabras o insinuaciones. En el caso de pacientes con demencia, según evaluaciones medicolegales, experimentan una reacción traumática aguda y expresan una confusión que puede imitar un empeoramiento de la cognición, incluso desencadenando recuerdos de abusos durante la infancia. En algunos casos, este tipo de maltrato es un precursor de mortalidad y muerte temprana $(19,21,22)$.

El abuso patrimonial o financiero especifica el mal uso, explotación o apropiación de los bienes por parte de terceros, sin consentimiento del anciano, siendo las personas con deterioro cognitivo las de mayor riesgo $(18,23)$.

La negligencia es el incumplimiento de las funciones propias del cuidador y se reconocen la activa y la pasiva. La primera ocurre cuando el victimario, por prejuicio o descuido, deja de proveer al adulto los cuidados de forma deliberada e intencionada, y la segunda es un descuido involuntario que sucede por ignorancia $(18,19,24)$. Los que cometen este tipo de maltrato pueden incurrir en el abandono, con lo que se desampara de manera voluntaria al anciano, generalmente dependiente. El 9\% de las personas mayores sufre maltrato por negligencia sobre todo en los ubicados en zonas rurales distantes (25). Dentro de este grupo surge la autonegligencia, como un tipo de maltrato que se manifiesta con la negación para obtener una adecuada alimentación, vestuario, vivienda, seguridad, higiene personal y medicación, que incluso puede llegar a un suicidio pasivo en un anciano mental y físicamente competente (18).

Además de los tipos clásicos de maltrato, la OMS y la INPEA incluyen otros dos tipos que involucran a la sociedad (26): el maltrato estructural, ejercido desde los gobiernos e instituciones, entendido como la discriminación en las políticas hacia los ancianos, la falta de recursos para cubrir las necesidades asistenciales, así como la necesidad de ingresos y de alojamiento, lo cual se considera que origina otros tipos de maltrato (18). En Colombia, en 2016, los hogares más pobres correspondían al $69,8 \%$, en los que viven los adultos mayores, y el $58,7 \%$ de los ancianos vive en un hogar donde los ingresos familiares no superan el medio salario mínimo al mes. Solo el $24,5 \%$ de las personas mayores tiene servicio de salud y ahorro para su pensión y el $70,2 \%$ trabaja de forma independiente (27).

Un estudio realizado con 4215 adultos mayores de 60 años en el departamento de Antioquia, el 45,2\% no recibió ingresos económicos en el mes anterior a la encuesta (28). Hay características propias del contexto colombiano que, por su historia y las condiciones sociodemográficas, hacen un diferencial, como es descrito en el estudio "Las personas viejas en Colombia: Memoria viva de las violencias y de las resistencias" (4), en el que destaca cómo las condiciones de adversidad durante el curso de la vida (en lo que respecta al conflicto armado y a las violencias restantes) han forjado una representación social y su interpretación de la realidad y de lo que para cada quien se interpreta como maltrato o violencia. Estas condiciones de vulnerabilidad son particulares de sectores poblacionales como estratos más bajos 
y condiciones de pobreza, lo que de por sí ya atribuye mayor riesgo de maltrato. Según la encuesta $\mathrm{SABE}$ el $15,4 \%$ de los que refirieron haber sido desplazados por conflicto armado o violencia reportó maltrato.

El otro tipo de maltrato es el social, asociado con los prejuicios y los estereotipos sobre las personas mayores, esto es, la representación de "carga", ligada a una connotación de discapacidad y pérdida de autonomía, lo que se construye socialmente a partir del retiro de las actividades laborales hasta la dependencia funcional, y se traduce en repercusiones de la dinámica familiar, en cuanto al cuidado, actitudes irrespetuosas, maltrato verbal y emocional, es decir, una actitud social negativa hacia el anciano $(5,29)$.

\section{Factores de riesgo}

El maltrato al adulto mayor es un problema complejo que no puede atribuirse a una causa única, sino que corresponde a una interacción de factores $(30,31)$. A continuación se describen los factores de riesgo de maltrato, partiendo de las características propias del maltratado, el maltratador y el contexto social $(18,32)$.

\section{Factores de riesgo del maltratado}

Algunas circunstancias potencian que los ancianos sean víctimas. La mayoría de los estudios indican que las mujeres son más propensas que los hombres a ser víctimas de abuso (2,33). En el contexto colombiano, en particular (Encuesta SABE; figura 6), las mujeres presentaron mayor proporción que los hombres en todos los tipos de maltrato, lo que se relaciona con los hallazgos de otros estudios colombianos $(28,34)$.

Existe una brecha de género en los adultos mayores de América Latina. La violencia contra las mujeres es mayor, se reproduce intergeneracionalmente y está más marcada en aquellas que crecieron en ambientes machistas y en su niñez fueron subordinadas. En un estudio realizado en Manizales (Colombia) se encontró que la experiencia de violencia física ocurrida alguna vez en la vida en mujeres era del 18,3\% comparado con el 2,2\% en los hombres; la violencia psicológica de pareja, del 19,4\% en mujeres comparada con el 10,7\% de los hombres, y la violencia psicológica de familia, del 18,3\% en mujeres comparada con el $8,3 \%$ de los hombres (2).

En un estudio descriptivo transversal realizado en Antioquia, en 2012, se encontraron como factores de riesgo vivir en el área urbana, sufrir ansiedad, tener sintomatología depresiva y apoyo familiar inadecuado (28).

En otro estudio reciente realizado en Pasto (Colombia), los principales factores asociados fueron dependencia, disfunción familiar, malos tratos entre miembros de la familia y escaso apoyo social (35). Así, las víctimas de abuso tienen menos contacto social; por lo tanto, sus relaciones sociales son menos satisfactorias (18).

Un factor de riesgo que se destaca en las revisiones es la dependencia, ya que a medida que esta aumenta, el cuidado incrementa la carga y estrés del cuidador (19). En el estudio de Agudelo-Cifuentes et al., las personas mayores con dependencia tienen siete veces más probabilidad de maltrato que los adultos mayores independientes (35).

Diversos estudios han encontrado que individuos con deterioro de la salud son más propensos a ser abusados $(18,36,37)$. El deterioro cognitivo y las alteraciones de la conducta como agresividad, agitación, deambulación y depresión son factores de riesgo para la explotación financiera (19).

Muchos de los factores de riesgo para el abuso y la negligencia son semejantes para el autoabandono. El 50\% de los casos se presenta en personas mayores de 80 años (38), con dependencia funcional, abuso de alcohol o drogas, aislamiento y enfermedad psiquiátrica (39). Otros factores de riesgo son la dependencia financiera y emocional, bajo nivel educativo, pobreza y viudez (40).

El maltrato tiene muchas consecuencias. Se ha documentado mayor riesgo de hospitalización y muerte $(19,41)$. El Estudio Internacional de Movilidad en Ancianos es un estudio 
longitudinal de base poblacional realizado en cinco ciudades, una de ellas Manizales, con el objetivo de entender la disminución de la movilidad y su asociación con circunstancias peculiares del entorno, como la pobreza, las diferencias de género y la violencia. Se encontró asociación entre la violencia física (en la infancia, por la familia o por la pareja) con la diminución de la movilidad y mayor riesgo de discapacidad; en cuanto a la violencia psicológica, está relacionada con mayor riesgo de depresión (42). En el estudio de Cano et al. (3), las personas mayores que han sufrido desplazamiento tienen mayor riesgo de enfermedades de larga duración que afectan su calidad de vida y las tasas de mortalidad por violencia.

\section{Factores de riesgo del que ejerce maltrato}

Es importante identificar los potenciales abusadores para poder prevenir el maltrato y, así, establecer la relación entre el agresor y la víctima en el continuo de la violencia intrafamiliar o en las instituciones de larga estancia. Según el National Elder Abuse Incidence Study, en el contexto de la violencia intrafamiliar, el maltrato se origina cuando lo comete uno de sus miembros, que suele ser el cuidador principal, entre los que se encuentran los hijos (50\%) y los cónyuges $(20 \%$ a $40 \%)$, de edades entre 40 y 59 años, y es más probable que sean hombres, que tengan un historial de abuso de sustancias, problemas de salud mental o física, antecedentes judiciales y que dependan económicamente de la víctima $(14,36,40,43)$. Los tipos más frecuentes de maltrato en el ámbito familiar son el psicológico, negligencia, abandono y abuso patrimonial $(31,44)$. En particular, vivir con un mayor número de miembros del hogar que no sea un cónyuge se asocia con un mayor riesgo de abuso, especialmente de tipo financiero y físico $(19,40)$.

Según la teoría del aprendizaje social, las conductas violentas son mecanismos aprendidos durante la infancia para expresar la frustración y la ira. De este modo, los sujetos que fueron maltratados probablemente se convertirán en maltratadores en su vida adulta, por lo que la experiencia familiar de abuso a personas mayores o violencia intrafamiliar previa son factores de riesgo importantes (18).

El cuidador desempeña un papel protagónico en la vida del anciano con algún grado de dependencia (18), lo que podría llevar a situaciones de estrés y frustración, la inadecuada formación en los cuidados básicos geriátricos o la ausencia de apoyo y falta de acceso a información de programas sociales pueden incrementar su sobrecarga, y por lo tanto existe mayor probabilidad de abuso financiero, emocional, físico y negligencia $(40,45)$.

Otros factores del que ejerce el maltrato y que pueden estar involucrados son la depresión, el abuso de alcohol, fármacos o drogas, las enfermedades mentales, los trastornos de personalidad, el aislamiento social y la incapacidad del cuidador para soportar emocionalmente los cuidados $(18,19)$. Desde la experiencia en investigaciones forenses y tratamiento a las víctimas, Ramsey-Klawsnik (46) propuso cinco tipos de abusadores en la tabla 1.

\section{Tabla 1}

Características de abusadores según el tipo de maltrato

\begin{tabular}{|c|c|c|}
\hline $\begin{array}{l}\text { Tipo de } \\
\text { abusador }\end{array}$ & Características & \begin{tabular}{|l|}
$\begin{array}{l}\text { Tipo de } \\
\text { maltrato }\end{array}$ \\
\end{tabular} \\
\hline Sobrecargado & $\begin{array}{l}\text { Individuos bien intencionados y cualificados para } \\
\text { prestar la atención, pero cuando las necesidades los } \\
\text { sobrepasan actúan de forma violenta. }\end{array}$ & Verbal o físico. \\
\hline Discapacitado & $\begin{array}{l}\text { Individuos con limitaciones fisicas o mentales para } \\
\text { brindar una adecuada atención y que pueden no ser } \\
\text { conscientes de ellas. }\end{array}$ & Negligencia. \\
\hline Narcisista & $\begin{array}{l}\text { Ven la relación como un medio para obtener un fin } \\
\text { y pueden ser atraídos por los hogares de ancianos o } \\
\text { centros donde pueden entrar en relaciones de } \\
\text { cuidado con los adultos vulnerables para satisfacer } \\
\text { sus propias necesidades. }\end{array}$ & $\begin{array}{l}\text { Financiero y } \\
\text { negligencia. }\end{array}$ \\
\hline Dominante & $\begin{array}{l}\text { Ejercen el poder y la autoridad. A menudo sienten } \\
\text { que la víctima merecía el maltrato. }\end{array}$ & $\begin{array}{l}\text { Negligencia, } \\
\text { maltrato } \\
\text { financiero y } \\
\text { sexual. } \\
\end{array}$ \\
\hline Sádico & $\begin{array}{l}\text { Personas que suelen tener un trastomo de } \\
\text { personalidad antisocial y obtienen placer realizando } \\
\text { actos violentos. }\end{array}$ & Maltrato físico. \\
\hline
\end{tabular}

\section{Factores de riesgo del entorno}

Se ha planteado que el maltrato hacia las personas mayores puede ser ejercido por la propia sociedad. Del contexto sociohistórico dependen los imaginarios colectivos de la vejez y el 
maltrato. En el caso de Colombia, el conflicto armado, caracterizado por violentos procesos de desplazamiento, tuvo un impacto negativo en las familias colombianas; para 1970 la mitad de la población se encontraba en situación de pobreza y la mayoría se asentaba en zonas rurales, características que se han mantenido hasta el día de hoy. El 26\% de la población del país es rural, el $62,1 \%$ de esta es pobre y el $21,5 \%$ está en la indigencia. En la encuesta SABE se encontró que las personas que tuvieron que desplazarse por violencia a la largo de la vida fueron el 8,65\%. Del total de desplazados, el 54,1\% tenía 70 o más años de edad y el 45,9\% tenía entre 60 y 69 años (3). En una sociedad en la que predomina una imagen negativa y estereotipada de la vejez, se manifiesta por el distanciamiento y la marginación (18).

Las instituciones dirigidas a la atención de adultos mayores, como establecimientos de larga estancia, centros de atención primaria de salud y hospitales, son entornos susceptibles de presentar casos de maltrato, a consecuencia de la falta de capacitación del personal, la sobrecarga del trabajador y la discriminación $(47,48)$. Los tipos de maltrato institucional son la infantilización, que limita su capacidad de autonomía; la despersonalización, donde se proporcionan servicios de acuerdo con criterios generales que desatienden sus necesidades individuales; la deshumanización, donde se desdeña su intimidad y autonomía; la victimización, con la que se ataca la integridad física y moral de la persona mayor mediante amenazas, intimidación y agresiones verbales, y, finalmente, la negligencia en la que no se proveen los cuidados o supervisión necesarios de alimentación, vestuario, higiene o cuidados médicos que se requieran $(18,29,49)$.

Dentro de otros factores de riesgo del entorno se destacan: falta de respuesta a los procesos legales impuestos, ausencia de apoyo tanto familiar como institucional, hacinamiento $y$ dificultades económicas (13).

\section{Evaluación}

Reconocer los casos de maltrato al anciano es un reto, ya que las víctimas pueden ocultar los hechos, no ser capaces de articularlas por la afectación cognitiva, ser confundidas como parte de la comorbilidad, llevar a falsos negativos (por ejemplo, la presencia de fracturas atribuidas erróneamente a la osteoporosis) o lleva a falsos positivos (como hematomas espontáneos atribuidos erróneamente al maltrato físico en la evaluación) $(19,50)$.

Se han desarrollado escalas estandarizadas para detectar maltrato, como la Elder Abuse Suspicion Index (EASI), desarrollada en Montreal, compuesta por seis preguntas dirigidas a personas mayores sin deterioro cognitivo en el ámbito de la atención primaria. Se realizó una validación de esta escala en España, donde al aplicarla se logró una alta especificidad (95\%) y una baja sensibilidad $(67 \%)(51,52,53)$.

La Indicators of Abuse (IOA) es un instrumento de 15 ítems sobre la persona mayor y 12 ítems sobre el cuidador. Discrimina entre abuso y no abuso, y se creó para que lo apliquen servicios sociales o sanitarios. Cuenta con un gran poder discriminativo y su fiabilidad aumenta si es realizada por profesionales en trabajo social $(54,55)$.

La Hwalek Sengstock Elder Abuse Screening Test (HS-EAST) está diseñada para identificar maltrato en personas con demencia. Se compone de 15 preguntas directas y requiere un tiempo estimado de entre 5 y 10 minutos para su realización. Varios estudios han mostrado su baja fiabilidad, pues no tiene poder discriminativo para el tamizaje del abuso a las personas mayores, debido al gran número de falsos positivos, por lo que posteriormente se planteó una adaptación de la escala: la Vulnerability Abuse Screening Scale (VASS), con 12 ítems. Los estudios realizados indican una confiabilidad interna de moderada a buena para la identificación de la dependencia, mas no para la detección de situaciones de maltrato (55). La Escala de Detección del Maltrato por parte del Cuidador (Caregiver Abuse Screen [CASE]) cuenta con 6 ítems dirigidos a los cuidadores; es un instrumento sensible y específico que puede utilizarse como tamizaje; pero debe complementarse con una valoración más profunda (56). Las escalas EASI y la IOA detectan en mejor medida a los ancianos 
maltratados sin deterioro cognitivo, y la CASE, en el caso de aquellos con deterioro cognitivo. Esta última se validó en 2015 en España; sin embargo, ninguna se ha validado en la población colombiana (55).

En esta revisión se proponen rutas de atención en caso de sospecha de maltrato a la persona mayor en la comunidad, abordado en atención primaria o en el servicio de urgencias (algoritmos 1 y 2). En las instituciones de salud, la atención de un caso de maltrato o con sospecha se puede dar por cualquiera de las vías de atención: urgencias, hospitalización y consulta externa (57), y todo caso se considera prioritario para recibir la atención. Es importante el manejo integral y multidisciplinario, a través del equipo de salud tratante y los grupos establecidos en cada institución, para el abordaje puntual de la situación de maltrato y sus factores de orden psicosocial. El diagnóstico incluye la evaluación inicial, la detección de problemas y necesidades, las hipótesis sobre el caso, el diseño de objetivos, la recolección de datos y las conclusiones. En la evaluación inicial, si lo considera pertinente, el médico debe diligenciar el consentimiento informado del paciente y verificar que no está en riesgo inminente su vida. Luego, procederá a realizar el interrogatorio y el examen físico completo. Su función más importante será reconocer e identificar los signos de maltrato, familiarizarse con los recursos disponibles en la comunidad local, derivar al paciente y coordinar las intervenciones con esos recursos $(19,52)$.

Los sospechosos de ser víctimas o victimarios deben ser entrevistados por separado en un ambiente comprensivo y exento de prejuicios. Inicialmente, al encontrarse frente a una víctima potencial, las preguntas indirectas suelen ser menos amenazantes. Algunos ejemplos son: ise siente seguro en su hogar? ¿Alguien maneja sus cuentas bancarias?, o en casos difíciles podrían ser necesarias las preguntas directas como: ien su hogar alguien lo lastima? Cuando usted necesitaba ayuda ialguien no lo ayudó? (19).

Para detectar oportunamente situaciones de maltrato y activar los recursos judiciales pertinentes es necesario reconocer los signos de alarma de todos los tipos de maltrato contra el adulto mayor. Hay algunos patrones emergentes en el anciano que son útiles para alertar al médico sobre la posibilidad de abuso que se nombran en la tabla 2 y no deben considerarse diagnósticos confirmados sin otros hallazgos clínicos o información de la anamnesis que lo corroboren. El abuso patrimonial y la negligencia son particularmente más difíciles de abordar por las manifestaciones más sutiles, lo que hace que la detección y la intervención sean críticas $(19,45)$. La negligencia se hace evidente con el aumento de la dependencia, por lo que es necesario evaluar si el cuidador ha cumplido con las necesidades del anciano según su funcionalidad. Una exploración detallada de los registros médicos previos puede revelar signos de maltrato que no se detectaron en el momento, pero que en retrospectiva indican maltrato (57).

\section{Tabla 2}

Signos de alarma de maltrato

\begin{tabular}{|c|c|}
\hline Tipo & Signos de alarma \\
\hline $\begin{array}{l}\text { Maltrato físico } \\
(19,63)\end{array}$ & $\begin{array}{l}\text { - Hematomas en la cara, aspecto lateral del brazo derecho y torso } \\
\text { posterior, incluidas las regiones posterior, pectoral, lumbar y glútea } \\
\text { - Abrasiones (rasguños) } \\
\text { - Fracturas } \\
\text { - Marcas de ataduras de muñeca } \\
\text { - Ausencia de pelo (alopecia traumática) } \\
\text { - Desprendimiento de retina }\end{array}$ \\
\hline $\begin{array}{l}\text { Maltrato } \\
\text { psicológico } \\
\text { (14) }\end{array}$ & $\begin{array}{l}\text { - Depresión, ansiedad y otras formas de angustia psicológica, en las } \\
\text { que normalmente se prescriben intervenciones farmacológicas y } \\
\text { psicoterapéuticas, que no remitirán a menos que se detecte y mitigue } \\
\text { el abuso } \\
\text { - Confusión y desorientación, paranoia, depresión y ansiedad } \\
\text { - Miedo a los extraños y a su ambiente natural } \\
\text { - Miedo a los cuidadores } \\
\text { - Baja autoestima } \\
\text { - Indecisión para hablar abiertamente } \\
\text { - Búsqueda de atención y afecto } \\
\text { - Cambio de actitud cuando está presente el potencial agresor }\end{array}$ \\
\hline $\begin{array}{l}\text { Maltrato } \\
\text { estructural } \\
\text { social }\end{array}$ & - Discriminación, marginalidad y exclusión social \\
\hline $\begin{array}{l}\text { Abuso sexual } \\
(19,21,22,64)\end{array}$ & $\begin{array}{l}\text { - Dolor genital o anal } \\
\text { - Hematomas perineales } \\
\text { - Enfermedades venéreas o infecciones genitales sin explicación } \\
\text { - Hemorragias vaginales o anales sin explicación } \\
\text { - Ropa interior rasgada, manchada o con sangre }\end{array}$ \\
\hline $\begin{array}{l}\text { Abuso } \\
\text { patrimonial } \\
(40)\end{array}$ & $\begin{array}{l}\text { - Patrón irregular de gastos o retiro de dinero de las cuentas } \\
\text { - Otorgamiento de poder a terceros para cobro de pensión } \\
\text { - Cambios repentinos al testamento } \\
\text { - Falta de utensilios de higiene personal y vestuario } \\
\text { - Cuesarición inexplicable de fondos o posesiones valiosas } \\
\text { - Evidencia de podas, cuando se supone que alguies materiales a cambio de cuidados pagando } \\
\text { - Desconocimiento del estado económico propio y la cesión de bienes, } \\
\text { muebles o inmuebles a un tercero familiar o no }\end{array}$ \\
\hline
\end{tabular}


Tabla 2 (cont.)

Signos de alarma de maltrato

\begin{tabular}{|l|l|}
\hline Negligencia & - Lesiones por presión \\
& - Malnutrición \\
& - Deshidratación \\
& - Descuido en la higiene personal y en el vestuario \\
& - Impactación fecal \\
& - Abandono de la persona en la cama, en la calle o en algún servicio \\
público & - Deterioro progresivo de la salud \\
& - Aislamiento \\
& - Mala adherencia a tratamientos médicos y el número de \\
& medicamentos excesivo o insuficiente en relación con los problemas \\
& de salud \\
& Desvinculación al sistema de salud \\
\hline
\end{tabular}

\section{Grupo multidisciplinario}

Los equipos multidisciplinarios pueden realizar una mejor interacción con la víctima y el agresor, desarrollar y coordinar las valoraciones continuas y periódicas y establecer relaciones más fuertes con la comunidad, con los responsables locales y con los organismos más capaces de proporcionar apoyo y orientación (45). Cada equipo debe estar adaptado a los recursos disponibles, el entorno y a la población objetivo específica. Un ejemplo sobresaliente de equipo de intervención es el Grupo de Buen Trato en el Hospital Universitario San Ignacio en Bogotá (Colombia), un grupo de naturaleza psicosocial, en el que inicialmente interviene psicología y trabajo social y, según el grupo poblacional, otras especialidades, como medicina de urgencias, geriatría, psiquiatría, pediatría y enfermería; incluso, en casos específicos pueden participar profesionales en derecho (57). En este caso, se han desarrollado guías institucionales como La guía de violencia intrafamiliar del Hospital Universitario San Ignacio, para la atención al adulto mayor víctima de maltrato, que facilita la identificación y atención apropiada a la persona mayor (58).

La valoración geriátrica integral es la herramienta del médico geriatra, útil en este ámbito para identificar el impacto global de todos los tipos de maltrato, las consecuencias y efectos que tiene sobre la persona mayor, además de prevenir el desarrollo de conductas que alteren el funcionamiento clínico, mental, funcional y social $(14,45)$.

Teniendo en cuenta el efecto negativo que genera el maltrato en los adultos mayores y las personas que las rodean, se considera de vital importancia brindar atención psicosocial oportuna para amortiguar la crisis desencadenada. La intervención psicosocial tiene como objetivos determinar si el abuso se ha producido o no y validar el testimonio después de la intervención de otros profesionales que también lo han recogido, tratamiento en casos en que el abuso ya se ha comprobado y la observación en casos dudosos (18).

La intervención del profesional de trabajo social en los casos de maltrato tiene como finalidad abordar la problemática, por una parte, identificando los factores desencadenantes de la situación y las consecuencias individuales y familiares; por otra, articulando las redes de apoyo disponibles para garantizar el restablecimiento de derechos vulnerados en la víctima (18).

\section{Coordinación interinstitucional}

La coordinación interinstitucional es el proceso por medio del cual se ordenan las funciones institucionales gubernamentales y no gubernamentales, a la vez que permite agilizar los trámites inherentes a los casos de acuerdo con los lineamientos dados por la normatividad vigente (59). Bajo coordinación interinstitucional, se debe realizar un plan de intervención a nivel individual, familiar y comunitario, dando lugar al proceso de reporte a los entes de control (comisarías de familia, Fiscalía General de la Nación) o seguimiento, si ya existen notificaciones previas, según la particularidad de cada caso, y se debe garantizar el reporte de estos al Sistema de Vigilancia Epidemiológica (Secretaría Distrital de Salud). El acompañamiento en este grupo poblacional es necesario, ya que se espera que con el envejecimiento poblacional las cifras de maltrato contra las personas adultas mayores se incrementen. Entonces, es preciso protegerlos jurídicamente, crear rutas para denunciar violaciones y hacer visible el problema a la sociedad (44). 


\section{Instancias legales}

En América Latina, los valores y creencias del colectivo pueden fomentar mayor tolerancia a la violencia en comparación con las sociedades más individualistas (34). Colombia es una sociedad cada vez más envejecida con antecedentes históricos importantes y un contexto cultural particular. Por ello, es necesario conocer las estrategias legales para la actuación frente a situaciones de maltrato.

Los esfuerzos más importantes en el mundo para fortalecer proteger los derechos en las personas ancianas son la Segunda Asamblea Mundial de Envejecimiento, de las Naciones Unidas, y la Segunda Conferencia Regional Intergubernamental sobre Envejecimiento de América Latina y el Caribe, realizada en Brasilia en 2007, que posicionaron el maltrato al anciano como uno de los problemas centrales para colocar en las agendas de los gobiernos, mediante la elaboración de estrategias sobre el fortalecimiento de los derechos humanos de las personas ancianas.

También está el Plan Internacional de Madrid sobre Envejecimiento y la Declaración de Toronto de 2002, que reconocen la deficiencia en la legislación alrededor de este tema, lo que hace necesario la solidaridad intergeneracional y el desarrollo de las estructuras que permitan la provisión de servicios (sanitarios, sociales, de protección legal, policiales, etc.) para responder de forma adecuada el problema $(10,60)$.

En el país, en 1975 se promulgó la Ley 29 para la protección a la ancianidad desprotegida, reglamentada por el Decreto 2011 de 1976, por el cual se organiza la protección nacional a la ancianidad. La dignidad humana es el eje central del Estado social de derecho y

[...] el Estado debe propender por el cuidado de la vejez como parte del cumplimiento de sus obligaciones constitucionales. Ello por cuanto a pesar de existir un especial deber de solidaridad en cabeza de la familia, el artículo 46 habla de una responsabilidad concurrente, y por tanto, el Estado no solo puede sino que debe contar con una política pública de cuidado, protección e integración del adulto mayor, y adoptar las respectivas medidas para implementarlas. (61)

El ordenamiento jurídico colombiano cuenta con la regulación que se resume en la tabla 3 . La Corte Constitucional establece el concepto de vejez, que debe definirse no como una enfermedad, sino como el deterioro natural, por lo cual se debe incrementar la inclusión social y la protección especial, debido a que eventualmente todos los seres humanos envejecen, y no es posible que exista discriminación y marginación por el hecho de la edad.

Tabla 3

Regulación del ordenamiento jurídico colombiano

\begin{tabular}{|l|l|}
\hline Ordenamiento jurídico colombiano & \multicolumn{1}{|c|}{ Contenido } \\
\hline $\begin{array}{l}\text { Constitución Política de Colombia } \\
1991\end{array}$ & $\begin{array}{l}\text { Artículo 13 } \\
\text { Artículo 46 }\end{array}$ \\
\hline Ley 687 de 2001 & Centros de bienestar del anciano \\
\hline Ley 700 de 2001 & $\begin{array}{l}\text { Por medio de la cual se dictan medidas tendientes } \\
\text { a mejorar las condiciones de vida de los } \\
\text { pensionados y se dictan otras disposiciones }\end{array}$ \\
\hline Ley 882 de 2004 & $\begin{array}{l}\text { Por medio de la cual se incrementan las penas en } \\
\text { caso de que el maltrato sea en contra de una } \\
\text { persona mayor }\end{array}$ \\
\hline Ley 1091 de 2006 & Colombiano y colombiana de oro \\
\hline Ley 1171 de 2007 & Beneficios a las personas adultas mayores \\
\hline Ley 1251 de 2008 & $\begin{array}{l}\text { Normas en procura de la protección, promoción y } \\
\text { defensa de los derechos de los adultos mayores }\end{array}$ \\
\hline Ley 1276 de 2009 & $\begin{array}{l}\text { Nuevos criterios de atención integral del adulto } \\
\text { mayor en los centros vida }\end{array}$ \\
\hline Ley 1315 de 2009 & $\begin{array}{l}\text { Condiciones mínimas que dignifiquen la estadía de } \\
\text { los adultos mayores en los centros de protección, } \\
\text { centros de día e instituciones de atención }\end{array}$ \\
\hline Ley 1850 de 2017 & $\begin{array}{l}\text { Establece medidas de protección al adulto mayor } \\
\text { en Colombia, se modifican las leyes 1251 de 2008, } \\
1315 \text { de 2009, 599 de 2000 y 1276 de 2009, se } \\
\text { penaliza el maltrato intrafamiliar por abandono y se } \\
\text { dictan otras disposiciones }\end{array}$ \\
\hline
\end{tabular}

\section{Seguimiento}

El seguimiento permite conocer en qué medida se han producido cambios en el paciente en la dirección de los objetivos marcados y en las diferentes áreas de intervención. Permite obtener información sobre el caso luego del diagnóstico inicial, acerca de la continuidad del tratamiento médico-terapéutico sugerido, el impacto psicosocial del evento en la víctima y la oportunidad del acceso a los mecanismos legales y de justicia. En esta etapa se debe garantizar el restablecimiento de derechos, verificar la integridad física de la víctima, la existencia de una red efectiva para el apoyo en salud mental y la reincorporación con seguridad a la vida social, 
económica y del proyecto de vida de la persona (19).

Una vez garantizada la integridad y seguridad del paciente, consolidado el diagnóstico y realizadas las acciones en salud, justicia, protección y salud pública correspondientes, se procederá a cerrar el caso, verificando que el anciano está en tratamiento y seguimiento. De todas formas, debe verificarse que el adulto mayor y su familia hayan recibido la información adecuada y suficiente, los derechos que le asisten y asesoramiento (62).

\section{Conclusiones}

Aunque muchos de los patrones identificados en la literatura mundial y latinoamericana coinciden con los colombianos, existen algunos que son casi idiosincráticos, como el fenómeno del desplazamiento provocado por el conflicto armado. Es muy probable que, como se registra en otros países, sea escasa la notificación del maltrato y existan sesgos de información graves por las mismas condiciones de la población anciana y por el tipo de perpetradores, que en la mayoría de los casos son sus mismos familiares. Esto sugiere que se requieren otros abordajes de investigación, diferentes a las tradicionales encuestas, posiblemente de enfoque cualitativo, como los estudios etnográficos (figuras 7 y 8 ).

\section{Figura 7}

Abordaje de un caso de maltrato hacia la persona mayor en la comunidad

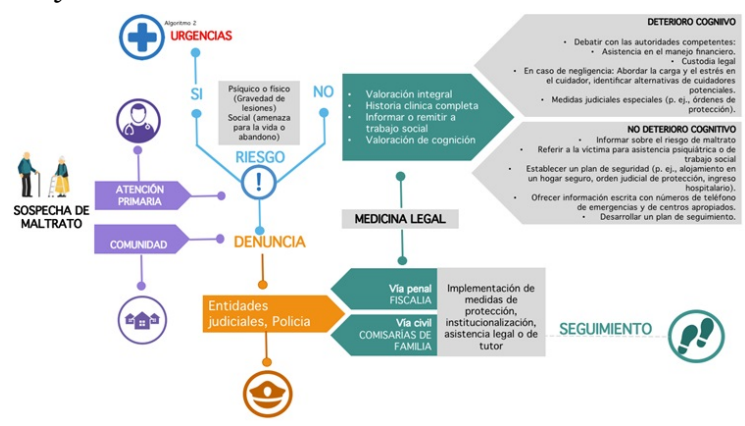

Fuente: adaptación del Plan de actuacio\#n en caso de maltrato, Organización Mundial de la Salud (OMS); Sociedad Española de Geriatría y Gerontología (SEGG, 2006).

Respuesta global al maltrato de las personas mayores incluyendo negligencia: capacitación de los servicios de atención primaria para el abordaje de un problema mundial.

\section{Figura 8}

Abordaje de caso de sospecha de maltrato hacia la persona mayor en el entorno hospitalario

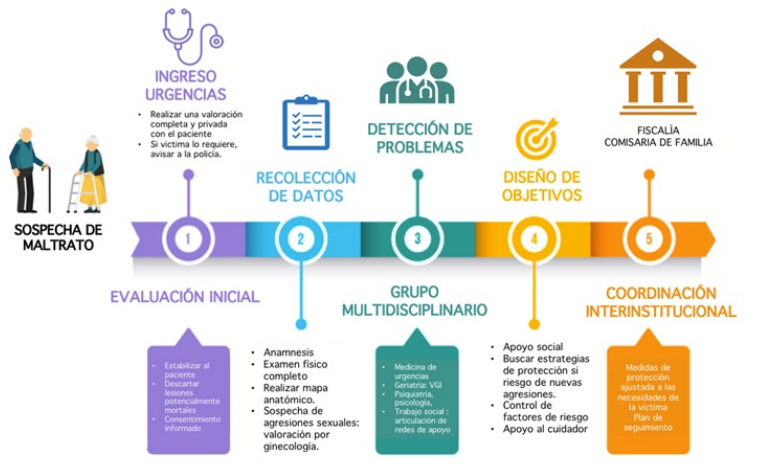

Las políticas públicas, aunque tienen un amplio componente en la legislación y la protección del Estado, deben comprometer la acción principal de la familia hacia sus deberes con el anciano y el reconocimiento de su rol fundamental en la sociedad humana. Es necesario un cambio cultural que lo reivindique y debe imprimirse en el curso vital humano desde edades muy tempranas.

Sobre todo, es importante evaluar el efecto de los programas de intervención y su seguimiento, el cumplimiento de las leyes que los protegen, así como la prevención del maltrato a través 
de la disminución de los factores de riesgo identificados como el género, la dependencia, la ruralidad, la pobreza y el desplazamiento por violencia, buscando incrementar la protección estatal, la disminución del machismo, la salud mental desde la infancia y el fortalecimiento de la paz.

\section{Referencias}

1. González Arana R, Molinares Guerrero I. La violencia en Colombia: Una mirada particular para su comprensión. Investig Desarroll [internet]. 2010;18(2). Disponible en: http://rcientificas.uninorte.edu.co/ind ex.php/investigacion/article/viewArtic le/1201/4599

2. Guedes DT, Alvarado BE, Phillips SP, Curcio CL, Zunzunegui MV, Guerra RO. The gender gap in domestic violence in older adults in Latin America: the IMIAS Study. Rev Panam Salud Publica. 2015;37(4-5):293-300.

3. Cano C, Borda M, Samper R, Medina M, Santacruz C. Las personas viejas en Colombia: Memoria viva de las violencias y de las resistencias. Rev Asoc Colomb Gerontol Geriatr [internet]. 2016;30. Disponible en: http://acgg.org.co/pdf/pdf_revista_ 16/articulo\%202\%20revista\%201-201 6.pdf

4. Ministerio de Salud de Colombia. SABE Colombia: Estudio Nacional de Salud, Bienestar y Envejecimiento. Bogotá: Ministerio; 2015.

5. Rueda MCA. Sentirse "una carga" en la vejez: realidad construida o inventada? Revista Kairós: Gerontologia. 2011;14(4):5-28.

6. Parales CJ, Ruiz ED. La construcción social del envejecimiento y de la vejez: Un análisis discursivo en prensa escrita. Rev Latinoam Psicol. 2002;1(1):107-21.
7. Marmolejo I. Violencia contra personas mayores. Barcelona: Ariel; 2005.

8. Tortosa JM. Personas mayores y malos tratos. Madrid: Pirámide; 2004.

9. Kessel H, Marín N, Maturana N, et al. Primera Conferencia Nacional de Consenso sobre el anciano maltratado. Rev Esp Geriatr Gerontol. 1996;31(6):367-72.

10. United Nations. Political Declaration and Madrid International Plan of Action on Ageing [internet]. Documento procedente del Second World Asembly of Aging; abril 8-12, 2002. Disponible en: https://www.un.org/en/events/past events/pdfs/Madrid_plan.pdf

11. Declaración de Toronto para la Prevención Global del Maltrato a las Personas Mayores. Rev Esp Geriatr Gerontol. 2002;37(6):332-3.

12. Moreno SL. Comportamiento de las lesiones de causa externa. Forensis. 2018;19(1):19-82.

13. Arellano P, Garreta B, Cervera A. Negligencia, abuso y maltrato. En: Tratado de geriatría para residentes. Madrid: Sociedad Española de Geriatría y Gerontología; 2006. p. 133-40.

14. Tinker A, Biggs S. The mistreatment and neglect of frail older people. En: Brocklehurst's textbook of geriatric medicine and gerontology (8th ed.). s. 1.: Elsevier; 2017. p. 939-44.

15. Yon Y, Mikton CR, Gassoumis ZD, Wilber KH. Elder abuse prevalence in community settings: A systematic review and meta-analysis. Lancet Glob Health. 2017;5(2):e147-e56.

16. Moya A, Barbero J. Malos tratos en personas mayores: marco ético. Rev Esp Geriatr Gerontol. 2003;38(3):129-88. 
17. Homer AC, Gilleard C. Abuse of elderly people by their carers. BMJ. 1990;301(6765):1359-62.

18. Arenas M, Mora T. Guía de prevención del maltrato a las personas mayores. Santiago de Chile: Senama; 2012.

19. Lachs MS, Pillemer KA. Elder Abuse. $\mathrm{N}$ Engl J Med. 2015;373(20):1947-56.

20. Organización Mundial de la Salud. Violencia contra la mujer: Violencia de pareja y violencia sexual contra la mujer. Washington: OMS; 2014.

21. Brasher K, Principle TD. Preventing sexual assault of older women: A framework for action. Los Angeles: OPAL Institute; 2017.

22. Speck PM, Hartig MT, Likes W, Bowdre T, Carney AY, Ekroos RA, et al. Case series of sexual assault in older persons. Clin Geriatr Med. 2014;30(4):779-806.

23. Lázaro M, Fernández AC, Serrano P, Benito MA, Villavicencio P, Santiago AE, Martín A. Abuso económico: Un tipo de maltrato en los pacientes mayores. Rev Esp Geriatr Gerontol. 2018;53 176-7.

24. del Carmen T, LoFaso VM. Elder neglect. Clin Geriatr Med. 2014;30(4):769-77.

25. Ministerio de Salud y Protección Social de Colombia. Día mundial de toma de conciencia contra el abuso y el maltrato en la vejez [internet]. 2018. Disponible en: https://www.minsalud.gov.co/Pagi nas/D\%C3\%ADa-mundialde-tomadeconciencia-contrael-abuso-maltratoen -lavejez.aspx.

26. Murphy K, Waa S, Jaffer H, Sauter A, Chan A. A literature review of findings in physical elder abuse. Can Assoc Radiol J. 2013;64(1):10-4.
27. Observatorio de la DemocraciaDepartamento de Ciencia Política de la Universidad de los Andes. La situación de los adultos mayores en Colombia. Bogotá; 2017.

28. Cano S, Garzo\#n MO, Segura AM, Cardona D. Factores asociados al maltrato del adulto mayor de Antioquia, 2012. Rev Fac Nac Salud Pu\#blica. 2015;33(1):67-74.

29. Fernández-Alonso MC, HerreroVelázquez S. Maltrato en el anciano: Posibilidades de intervención desde la atención primaria (I). Aten Prim. 2006;37(1):56-9.

30. Abusleme Lama MT, Guajardo Soto G, editores. El maltrato hacia las personas mayores en la Región Metropolitana, Chile: Investigación cualitativa en vejez y envejecimiento [internet]. Santiago de Chile: Senama; 2013. Disponible en: http://www.senama.gob.cl/storage/ docs/El-maltrato-hacia-las-personas-m ayores-en-la-RM-2013-investigacion-c ualitativa-en-vejez-y-envejecimiento-2 013.pdf

31. Daly JM. Evidence-based practice guideline: Elder abuse prevention. J Gerontol Nurs. 2018;37(11):11-7.

32. Martínez CG, Morante JJH, Martínez EC, Belzunce AG, Alarcón VN. Factores relacionados con el maltrato no institucional en residencias de personas mayores. Rev Esp Geriatr Gerontol. 2016;51:317-22.

33. Laumann EO, Leitsch SA, Waite LJ. Elder mistreatment in the United States: Prevalence estimates from a nationally representative study. J Gerontol B Psychol Sci Soc Sci. 2008;63(4):S248-s54.

34. Guedes DT, Alvarado BE, Phillips SP, Curcio CL, Zunzunegui MV, Guerra RO. Socioeconomic status, social relations and domestic violence 
(DV) against elderly people in Canada, Albania, Colombia and Brazil. Arch Gerontol Geriatr. 2015;60(3):492-500. 35. Agudelo-Cifuentes MC, Cardona Arango D, Segura Cardona A, Segura Cardona A, Muñoz Rodríguez D, Restrepo-Ochoa A. Características sociales y familiares asociadas al maltrato al adulto mayor de Pasto, Colombia 2016. CES Psicología [internet]. 2019;12(1):32-42. Disponible en: http: //revistas.ces.edu.co/index.php/psicolo gia/article/view/4387

36. Gorbien MJ, Eisenstein AR. Elder abuse and neglect: an overview. Clin Geriatr Med. 2005;21(2):279-92.

37. Tronetti P. Evaluating Abuse In The Patient With Dementia. Clin Geriatr Med. 2014;30(4):825-38.

38. Dong X. Elder self-neglect: Research and practice. Clin Interv Aging. 2017;12:949-54.

39. Dong $X$, Chen $\mathrm{R}$, Chang ES, Simon M. Elder abuse and psychological well-being: A systematic review and implications for research and policy-A mini review. Gerontology. 2013;59(2):132-42.

40. Peterson JC, Burnes DP, Caccamise PL, Mason A, Henderson CR Jr, Wells MT, et al. Financial exploitation of older adults: A population-based prevalence study. Journal of general internal medicine. 2014;29(12):1615-23.

41. Murphy SA, Osborne H, Smith I. Psychological consultation in older adult inpatient settings: A qualitative investigation of the impact on staff's daily practice and the mechanisms of change. Aging Ment Health. 2013;17(4):441-8.

42. Guedes D, Vafaei A, Alvarado BE, Curcio CL, Guralnik JM, Zunzunegui MV, Guerra RO. Experiences of violence across life course and its effects on mobility among participants in the International Mobility in Aging Study. BMJ Open. 2016;6(10):e012339.

43. The National Elder Abuse Incidence Study (NEAIS). National Center on Elder Abuse (NCEA), American Public Human Services Association (APHSA); 1998.

44. Pina E. Violencia intrafamiliar contra el adulto mayor en una comunidad de Guinea Bissau. Medisan. 2013;17(7):1053-9.

45. Montes C, Júnior P. Maltrato a los ancianos. En: Tratado de medicina geriátrica. Madrid: Elsevier; 2015.

46. Ramsey-Klawsnik H. Elder-abuse offenders: A typology. Generations. 2000;24(2):17-22.

47. Powers JS. Common presentations of elder abuse in health care settings. Clin Geriatr Med. 2014;30(4):729-41.

48. Radermacher H, Toh YL, Western D, Coles J, Goeman D, Lowthian J. Staff conceptualisations of elder abuse in residential aged care: A rapid review. Australas J Ageing. 2018;37(4):254-67. https://doi.org/10.1111/ajag.12565

49. Galnares TA. El maltrato en la vejez. Nuberos Científica. 2015;2(14).

50. Gibbs LM, Mosqueda L. Medical implications of elder abuse and neglect. Clin Geriatr Me. 2014;30(4):xv-xvi.

51. Yaffe MJ, Wolfson C, Lithwick M, Weiss D. Development and validation of a tool to improve physician identification of elder abuse: the Elder Abuse Suspicion Index (EASI). J Elder Abuse Negl. 2008;20(3):276-300.

52. Grupo de Trabajo de la Guía de Práctica Clínica sobre la Atención Integral a las Personas con Enfermedad de Alzheimer y Otras Demencias. Guía de práctica clínica sobre la atención integral a las personas con 
enfermedad de Alzheimer y otras demencias. Cataluña: Ministerio de Sanidad, Política Social e IgualdadAgència d'Informació, Avaluació i Qualitat en Salut de Cataluña; 2010.

53. Pérez-Rojo G, Izal M, Sancho MT, Bien GDIT. Adaptación lingüística y cultural de dos instrumentos para la detección de sospecha de maltrato hacia las personas mayores. Rev Esp Geriatr Gerontol. 2011;45 (4):213-8.

54. Reis M, Nahmiash D. Validation of the indicators of abuse (IOA) screen. Gerontologist. 1998;38(4):471-80.

55. Ugartondo Sanabria LV. Desvelando una realidad oculta: Detección y abordaje del maltrato intrafamiliar al anciano en atención primaria. Vizcaya: Universidad del País Vasco; 2017.

56. Cohen M, Halevi-Levin S, Gagin R, Friedman G. Development of a screening tool for identifying elderly people at risk of abuse by their caregivers. J Aging Health. 2006;18(5):660-85.

57. Rosen T, Stern ME, Elman A, Mulcare MR. Identifying and Initiating Intervention for elder abuse and neglect in the emergency department. Clin Geriatr Med. 2018;34(3):435-51. https://doi.org/10. 1016/j.cger.2018.04.007

58. ALMERA (Sistema de Gestión Integral) del Hospital Universitario San Ignacio, apartado de Gestión Asistencial Integral: Grupo de Buen Trato del Hospital Universitario San Ignacio. Procedimiento de atención integral a víctimas de maltrato al adulto mayor; 2015/03/16.

59. Atehortúa R. El reconocimiento de los derechos de los adultos mayores [disertación doctoral]. Bogotá: Universidad Nacional de Colombia; 2015.
60. Declaración de Brasilia: Segunda Conferencia Regional Intergubernamental sobre Envejecimiento en América Latina y el Caribe: hacia una sociedad para todas las edades y de protección social basada en derechos; 2011.

61. Sentencia C-503 de 2014 (magistrado ponente Jorge Ignacio Petrelt Chaljub), sobre criterios de atención integral del adulto mayor em centros de vida [internet]; 2014. Disponible en: http://www.corteconstitucional.go v.co/relatoria/2014/C-503-14.htm

62. del Nogal ML, Férnandez C, Serrano P, et al. Detección y seguimiento de malos tratos en personas mayores en un hospital terciario: experiencia de 3 años. Rev Esp Geriatr Gerontol. 2018;53:15-8.

63. Gibbs LM. Understanding the medical markers of elder abuse and neglect: Physical examination findings. Clin Geriatr Med. 2014;30(4):687-712.

64. Tzeng YL, Lin LC, Shyr YIL, Wen JK. Sexual behaviour of institutionalised residents with dementia - A qualitative study. J Clin Nurs. 2009;18(7):991-1001. 